\title{
Atomic-Scale Assessment of the Crystallization Onset in Silicon Carbonitride
}

\author{
Gabriela Mera* ${ }^{1}$ Ryo Ishikawa, ${ }^{2}$ Emanuel lonescu, ${ }^{1}$ Yuichi Ikuhara, ${ }^{2}$ Ralf Riedel ${ }^{1}$ \\ ${ }^{1}$ Technische Universität Darmstadt, Fachbereich Material- und Geowissenschaften, Jovanka- \\ Bontschits-Strasse 2, Darmstadt, D-64287, Germany; mera@materials.tu-darmstadt.de \\ ${ }^{2}$ Institute of Engineering Innovation, School of Engineering, The University of Tokyo, 2-11-16 \\ Yayoi, Bunkyo-ku, Tokyo 113-8656 Japan
}

\begin{abstract}
Within the present study, atomic-scale electron microscopy investigation on the crystallization behavior of polysilylcarbodiimide-derived SiCN was performed. The asprepared SiCN sample was found to be homogeneous and consisted of amorphous silicon nitride nano-domains dispersed within an amorphous, highly entangled graphene-like carbon matrix. Annealing of the sample at $1400{ }^{\circ} \mathrm{C}$ induced a slight increase of the ordering of the carbon phase. Additionally, the crystallization onset of the silicon nitride has been observed for the first time. In the sample annealed at $1400{ }^{\circ} \mathrm{C}$ small nano-clusters with average sizes of $0.8,1.5$ and $2.1 \mathrm{~nm}$ (consisting of 30,180 and 570 atoms, respectively; corresponding to $\mathrm{Si}_{12} \mathrm{~N}_{18}, \mathrm{Si}_{72} \mathrm{~N}_{108}$ and $\mathrm{Si}_{228} \mathrm{~N}_{342}$ ) were imaged and assigned to $\alpha$-silicon nitride. The crystallization of the amorphous silicon nitride phase into $\alpha-\mathrm{Si}_{3} \mathrm{~N}_{4}$ is thought to occur via diffusion of $\mathrm{Si}$ and $\mathrm{N}$, which rely on the presence of large number of dangling bonds in the amorphous SiCN sample.
\end{abstract}

Keywords: Silicon carbonitride, polymer-derived ceramics, phase separation, atomicresolution STEM, EELS

\section{Introduction}

(C) 2015. This manuscript version is made available under the Elsevier user license

http://www.elsevier.com/open-access/userlicense/1.0/

Page 1 of 15 
During the last decades, a continuously growing research interest has been given to amorphous ceramics particularly polymer-derived ceramics (PDCs), which are prepared via thermal treatment of suitable preceramic polymers. ${ }^{1-3}$ Owing to the strong covalent bonding and structural amorphous nature of PDCs, the ceramics in the ternary system Si-C-N as well as quaternary ceramics Si-M-C-N $(\mathrm{O})(\mathrm{M}=\mathrm{B}, \mathrm{Al}, \mathrm{Zr})$ have excellent structural stability, nearzero steady state creep as well as oxidation and corrosion resistance at high temperatures $\left(1500{ }^{\circ} \mathrm{C}\right), .^{1,4-7}$ Recently, numerous studies of SiCN ceramics have been reported on their amorphous structure and their high-temperature evolution over the ranges of micro to nanoscales. For understanding of the unusual stability of PDCs at high-temperatures, a wide variety of experimental techniques have been implemented such as X-ray and neutron diffractions, ${ }^{2-5}$ transmission electron microscopy ${ }^{6}$ and nuclear magnetic resonance. ${ }^{7}$ Moreover, theoretical approaches have also performed based on molecular dynamics, ${ }^{8-9}$ reverse Monte Carlo method, ${ }^{3}$ and density functional theory (DFT) calculations. ${ }^{10}$

Typical amorphous ceramics in the ternary Si-C-N system can be obtained from preceramic polymers such as polysilylcarbodiimides ${ }^{11}$ or poly(organyl)silazanes. ${ }^{12}$ The phase composition, physical or chemical properties and the microstructure of the resultant SiCN ceramics strongly depend on the used preceramic polymers. For example, polysilylcarbodiimide-derived $\mathrm{SiCN}$ materials are multi-phase materials consisting of amorphous $\mathrm{Si}_{3} \mathrm{~N}_{4}$, and segregated amorphous carbon phases. ${ }^{11,13}$ In contrast, polysilazanes can form homogeneous or single-phase amorphous ceramics, consisting of a glassy network based on $\mathrm{SiC}_{\mathrm{x}} \mathrm{N}_{4-\mathrm{x}}(0<\mathrm{x}<4)$ tetrahedra. ${ }^{13}$ Polysilazane-derived SiCN ceramics undergo phase separation at $1300^{\circ} \mathrm{C}$, leading to multiple-phase amorphous materials consisting of amorphous carbon, $\mathrm{Si}_{3} \mathrm{~N}_{4}$ and $\mathrm{SiC}^{13}$ To date this amorphous phase-separation is considered to occur without crystallization and being promoted by the evolution of hydrogen (small amounts of hydrogen are present in $\mathrm{SiCN}$ at temperatures up to $\left.1300^{\circ} \mathrm{C}\right)^{11}$ and the formation of dangling bonds which has been reported from electron paramagnetic resonance (EPR) spectroscopy measurements. ${ }^{14-15}$ Upon increasing the annealing temperature beyond $1500^{\circ} \mathrm{C}$, both polysilazane- and polysilylcarbodiimide-derived ceramics start to crystallize..$^{13}$ The crystallization of PDC-SiCN ceramics is believed to be promoted by the carbothermal decomposition of the amorphous silicon nitride nano-domains and thus to induce the formation of $\beta$-SiC (cf. a-Si $\mathrm{N}_{4}+3 \mathrm{C} \rightarrow 3 \beta-\mathrm{SiC}+2 \mathrm{~N}_{2} \uparrow$ ). ${ }^{12-13}$ However, the crystallization behavior in amorphous SiCN ceramics at high temperatures is still not well understood. The temperature depending crystallization and phase separation are thought to strongly depend not only on the chemistry of the precursors ${ }^{13,16-17}$ but also on the processing route, such as cross-linking and shaping, heating rate, carbon content and dwelling time. ${ }^{18}$ 
In the present study, we show the direct determination of the local atomic and chemical structural changes in amorphous SiCN ceramics upon annealing at high-temperatures (1100 and $1400{ }^{\circ} \mathrm{C}$ ), by using atomic-resolution scanning transmission electron microscopy (STEM). Through combining light-element sensitive annular bright-field (ABF) and atom-number $(Z)$ sensitive annular dark-field (ADF or Z-contrast) STEM imaging with electron energy-loss spectroscopy, we uncover the phase-separation and crystallization behavior of SiCN.

\section{Experimental Procedure}

Materials. Monolithic SiCN-based ceramic was obtained, as previously described, ${ }^{18}$ from poly(vinylphenylsilylcarbodiimide) via warm-pressing followed by pyrolysis in inert atmosphere at $1100^{\circ} \mathrm{C}$ (sample $\left.\mathrm{HN} 1-1100\right)$. The pyrolysis of the polymer was performed in a quartz Schlenk tube under a continuous flow of argon. Additionally, the sample HN1-1100 was annealed at $1400^{\circ} \mathrm{C}$ in a high-temperature graphite furnace (Thermal Technology Inc., CA USA) and using argon atmosphere to furnish sample HN1-1400. X-ray diffraction (XRD)

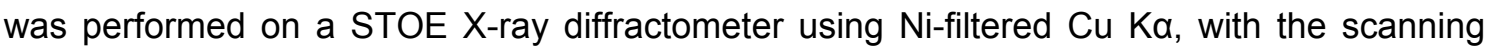
speed of $2 \theta$ of $7 \mathrm{~s}$ for $0.05^{\circ}$. Micro-Raman spectra were recorded with a Horiba HR800 microRaman spectrometer (Horiba Jobin Yvon, Bensheim, Germany) equipped with an $\mathrm{Ar}^{+}$laser (irradiation wavelength $514.5 \mathrm{~nm}$ ).

Electron microscope observations. The electron-transparent thin specimens were prepared by gently crushing the ceramic samples in ethanol and dispersed it onto perforated amorphous carbon. Note that we did not use the usual Ar-ion thinning method to avoid a significant surface damage. SAED patterns and BF-TEM images were taken with a $200 \mathrm{kV}$ JEM-2010HC electron microscope (JEOL). Atomic-resolution ABF/ADF-STEM observations were performed by $200 \mathrm{kV}$ aberration-corrected electron microscopes of JEM 2100F and JEM ARM-200CF (JEOL), equipped with a cold field-emission gun and a Gatan Enfina EELS spectrometer.

\section{Results and Discussion}

Figure 1a shows X-ray diffraction patterns obtained from polysilylcarbodiimide-derived SiCN annealed at $1100{ }^{\circ} \mathrm{C}(\mathrm{HN} 1-1100)$ and $1400{ }^{\circ} \mathrm{C}$ (HN1-1400). In both patterns, there is no distinct Bragg reflection but weak and broad peaks were found, suggesting that these specimens are amorphous in their nature. The broad bump centered at ca. $19^{\circ}$ was assigned to the 101 reflection of $\alpha$-silicon nitride; whereas that at ca. $45^{\circ}$ corresponds to the 100 
reflection of graphite. This features indicate that the SiCN samples investigated within this study might be described as consisting of an intimate mixture of silicon nitride and segregated carbon.
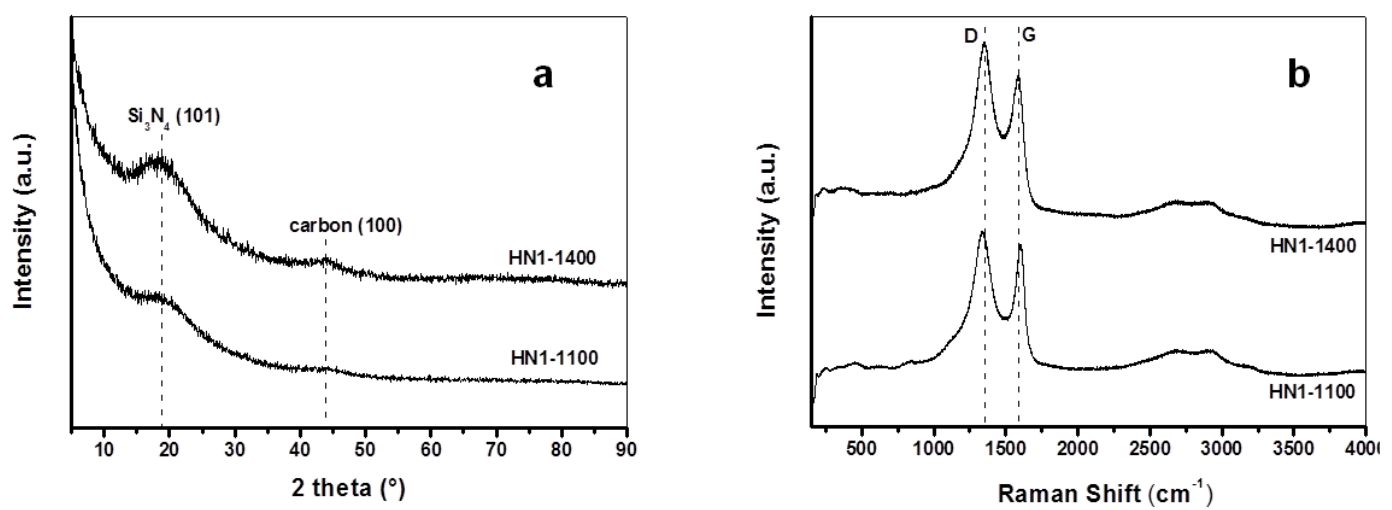

Figure 1. (a) X-ray diffraction patterns and (b) Micro-Raman spectra obtained from HN1-1100 and HN1-1400, respectively.

The micro-Raman spectra in Figure $1 \mathrm{~b}$ indicate the presence of disordered segregated carbon within the samples. On a basis of the relative peak intensity ratio of $I(D) / I(G)$, the in-plane (lateral) average size of clusters or graphene-like carbon nanodomains $^{19-20}$ are estimated to be $1.87 \mathrm{~nm}$ for the sample HN1-1100 and $2.43 \mathrm{~nm}$ for the sample HN1-1400 (Figure 1b). ${ }^{18}$ Furthermore, the so-called inter-defect distance $L_{D}$ (i.e., defects related to microporosity within the carbon phase, buckled graphene layers, punctual defect such as saturated $\mathrm{C} \mathrm{sp}{ }^{3}$ atoms as well as to the presence of dopants, such as nitrogen $)^{21}$ has been shown to increase from $6.49 \mathrm{~nm}$ in the sample prepared at 1100 ${ }^{\circ} \mathrm{C}(\mathrm{HN} 1-1100)$ to $7.47 \mathrm{~nm}$ in $\mathrm{HN} 1-1400 .{ }^{18}$ The evolution of both $\mathrm{L}_{\mathrm{a}}$ and $\mathrm{L}_{d}$ with temperature indicate that the ordering of the carbon phase increases as the annealing temperature is increased.

Despite the fact that the XRD patterns and the Raman spectra of both samples, HN1100 and HN1-1400, do not exhibit significant differences, the two samples are supposed to significantly differ from each other in terms of i) crystallization processes and ii) ordering of the segregated carbon phase. It is known that polymer-derived ceramics exhibit some nanoheterogeneity with respect to their network topology. Accordingly, mixed-bonds-containing $\mathrm{SiOC}$ or $\mathrm{SiCN}$ materials do not show a statistical distribution of their $\mathrm{SiO}_{x} \mathrm{C}_{4-\mathrm{x}} / \mathrm{SiC}_{x} \mathrm{~N}_{4-x}$ tetrahedra, but rather some clustering based on rearrangement processes, as previously described in the literature. ${ }^{22-23}$ 
Figures $2 \mathrm{~b}$ and $2 \mathrm{c}$ show representative selected-area electron diffraction (SAED) patterns obtained from samples $\mathrm{HN} 1-1100$ and $\mathrm{HN} 1-1400$, and the radial integrated intensity profiles are shown in Figure 2a. There are no Bragg reflections, but we found several DebyeScherrer rings as marked by arrowheads, suggesting that both specimens keep their amorphous nature even after annealing at $1400{ }^{\circ} \mathrm{C}$. Although the present electron diffractograms of these specimens have similar features, the Debye-Scherrer rings in HN11400 are more intense than those of $\mathrm{HN} 1-1100$, as it is evident from the radial integrated distribution profiles showing narrower peaks with higher intensities. These results are compatible with the X-ray diffraction patterns of Figure 1a. The two distinct peaks were identified as 110 and 120 reflections of graphite. Hence, the short-range-order (or the degree of crystallinity) of the carbon matrix in $\mathrm{SiCN}$ may be improved upon exposing the samples to high temperatures. Figures $2 \mathrm{~d}$ and $2 e$ show typical bright-field (BF) TEM images obtained from samples HN1-1100 and HN1-1400. As expected from the SAED pattern, sample HN11100 exhibits a homogeneous amorphous contrast and no precipitates were identified. While in sample HN1-1400 'jellyfish'-like contrasts were found in the BF-TEM image of Figure 2e (see also inset image of Figure 2e), indicating phase separation / segregation processes occurring in SiCN upon annealing at $1400{ }^{\circ} \mathrm{C}$. However, the size of the 'jellyfish'-precipitates is relatively small $(10-100 \mathrm{~nm})$ and the total volume of the precipitates is considerably smaller than that of the carbon matrix, and hence the chemical composition or the local structure of the precipitates from conventional X-ray or electron diffraction methods was impossible to identify. 


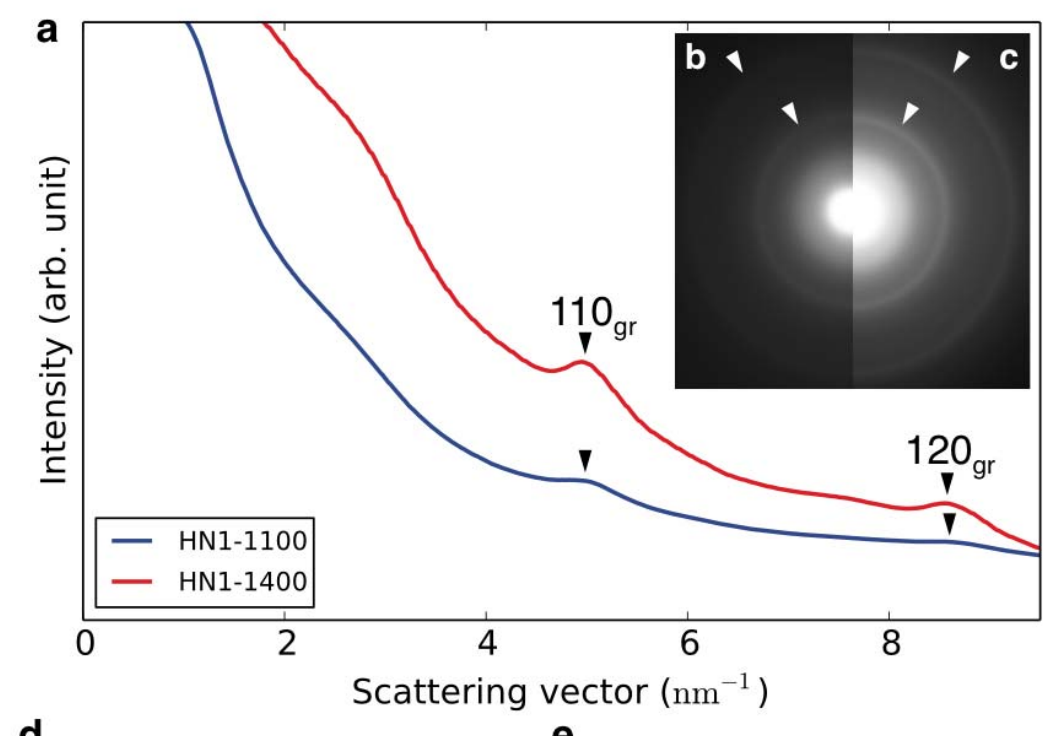

d

e
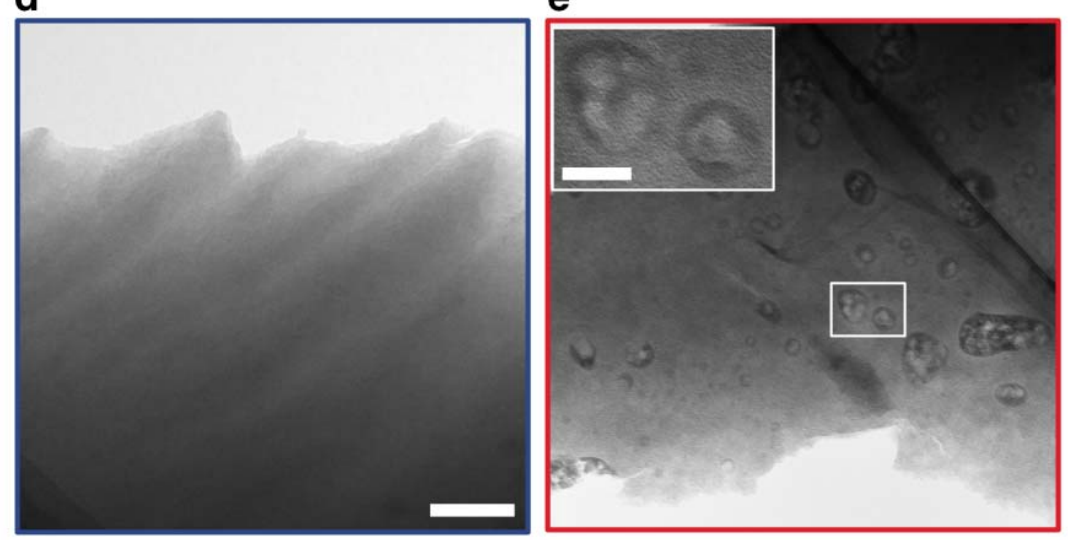

Figure 2. Radial integrated profiles (a) derived from SAED patterns obtained from (b) HN1-1100 and (c) HN1-1400. The indices of the Debye-Scherrer rings (arrowheads) correspond to the 110 and 120 reflections of graphite, respectively. BF-TEM images obtained from HN1-1100 (d) and HN1-1400 (e); the scale bar in all images is $100 \mathrm{~nm}$.

In order to elucidate the local structure and chemical composition in our polysilylcarbodiimide-derived SiCN ceramics, atomic-resolution ABF/ADF-STEM imaging was performed. Figures $3 a$ and $3 b$ show simultaneously recorded atomic-resolution ABF/ADF-STEM images obtained from sample HN1-1100. In the ABF image, curvedgraphene-layers which are entangled and which exhibit significant disorder (turbostratic structure) can be seen. The signal intensity of ADF-STEM image depends on the scattering power of the atoms (Z-contrast; $Z$ is atomic number) and therefore it is suitable to identify the locations of heavier constituent atoms, i.e., silicon atoms should appear as brighter contrasts in this case. On the other hand, recently developed ABF-STEM ${ }^{24}$ is significantly sensitive to the light elements including lithium ${ }^{25,26}$ and even hydrogen atoms ${ }^{27,28}$, but it has inverse contrast to ADF-STEM and the atoms such as carbon or nitrogen atoms can be seen as 
weak-dark contrast. Our microscope has two annular detectors and we can observe both light and heavy atoms by means of simultaneously recorded ABF/ADF-STEM images.

a

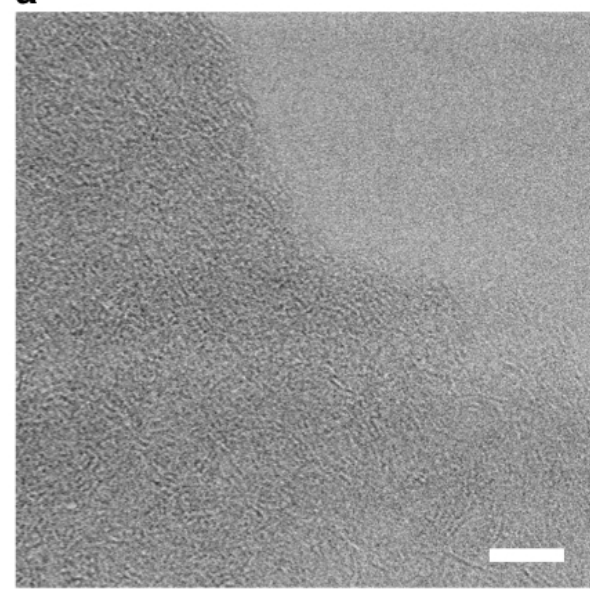

C

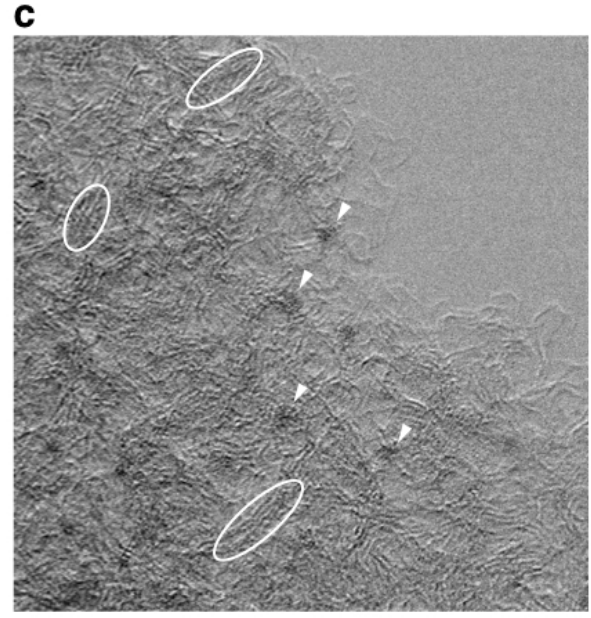

b

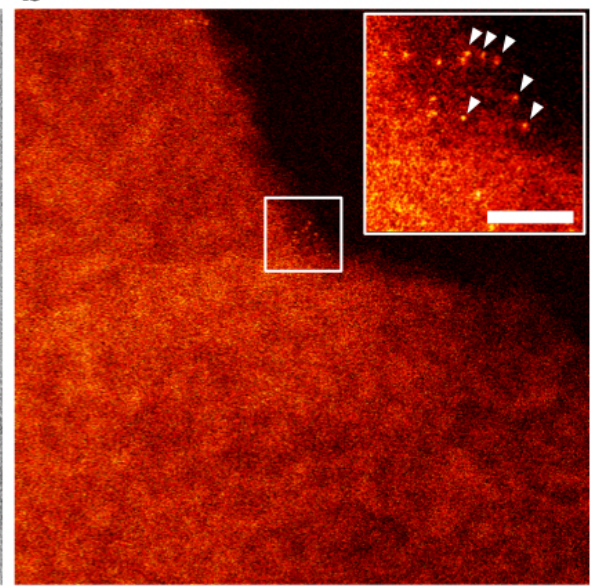

d

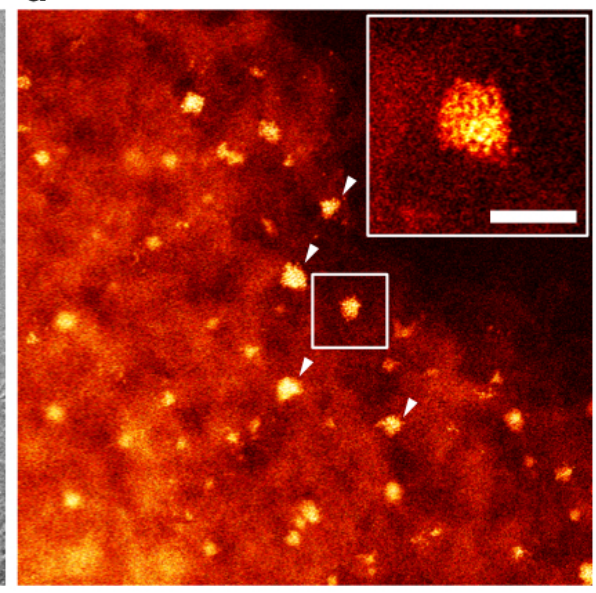

Figure 3. Simulatenously recorded ABF/ADF-STEM images obtained from HN1-1100 ( $a$ and $b$, resprectively) and HN1-1400 (c and d, respectively). The scale of the images (a) - (d) is the same and the scale bar in (a) is $5 \mathrm{~nm}$. The white-square regions are enlarged in the inset images (the scale bar in the insets is $2 \mathrm{~nm}$ ).

In the ADF image, the Z-contrast is relatively uniform but a number of brighter atoms distributed in the whole area has been found (see an enlarged inset image of Figure $3 b$ ), which, based on their Z-contrast nature, should be individual atoms. ${ }^{29}$ Upon repeating the scan of the electron probe at the same area, an electron-beam-induced movement of the individual Si atoms was observed, indicating that they are not strongly bonded to the carbon matrix but have a degree of freedom for the movement within the matrix. This result is in well agreement with EPR spectroscopy results reported in the literature, ${ }^{14-15}$ indicating the presence of Si dangling bonds in PDC ceramics. Figures $3 c$ and $3 d$ show ABF/ADF-STEM 
images obtained from a typical matrix region of sample HN1-1400. As expected from X-ray and electron diffraction, the bulk carbon structure in HN1-1400 shows the enhancement of a local structural order as fringe contrasts in ABF-STEM image (marked by ovals in Figure 3c); those fringes were not observed in sample HN1-1100. Moreover, a number of nano-clusters with dark contrast and 1-2 $\mathrm{nm}$ in size (marked by arrowheads in Figure 3c) have been found. These nano-clusters are also observed as brighter contrasts in the Z-contrast image of Figure $3 \mathrm{~d}$ and consequently consist of Si atoms.

The size-distribution of the Si-containing nano-clusters in sample HN1-1400 was investigated by extracting the local strong contrasts in the ADF-STEM image, where the cluster-shape was reasonably approximated as spherical. Figure 4a represents a lowmagnification Z-contrast image and the corresponding binary image is shown in Figure $4 \mathrm{~b}$ (white spots indicate the nano-clusters). On the basis of the particle analysis implemented in Image $\mathrm{J}^{30}$ the diameters of ca. 300 nano-clusters were assessed (size distribution shown in Figure 4c). Although the size of the clusters is broadly distributed, we can classify the cluster size distribution into three types with average values of $0.8,1.5$, and $2.1 \mathrm{~nm}$ in size, which were estimated by multiple-peak-fitting with three independent Gaussian profiles.

The composition of the nano-clusters formed in sample HN1-1400 was investigated by using EELS. The spectra were collected across the nano-clusters with $0.02 \mathrm{~nm}$ steps (measurements along the line between $X$ and $X$ ', see inset of Figure $4 d$ ). We found $S i-L_{2,3}, C-$ $\mathrm{K}$ and $\mathrm{N}-\mathrm{K}$ edges in all spectra and no other elements were detected. In order to construct a relative concentration map, the net edge signals were integrated over and the profiles are plotted in Figure 4d (the background was subtracted by a power-law fit). The carbon concentration gradually increases along the $X-X^{\prime}$ direction because the background of the carbon matrix becomes thicker. On the other hand, the silicon and nitrogen concentrations steeply increase and are localized at the nano-cluster region (see the Z-contrast profile), suggesting that the formed nano-clusters in sample HN1-1400 consist of silicon and nitrogen (note that these features were representative for the investigated sample and were reproduced for several different nano-clusters). 

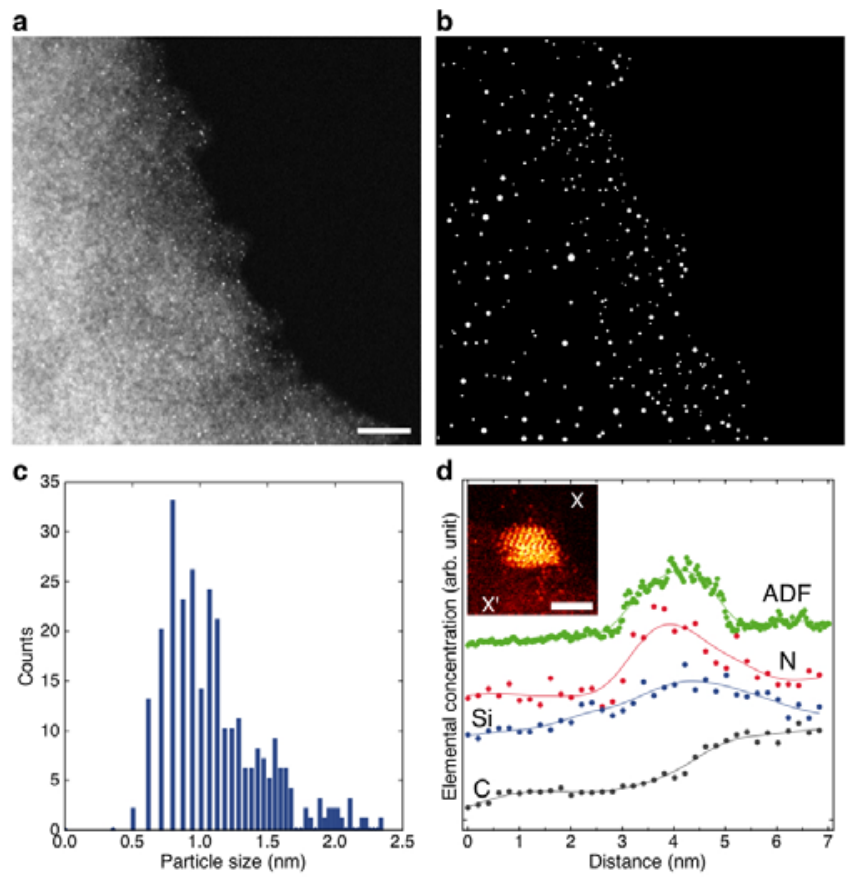

Figure 4. (a) Low-magnification Z-contrast image obtained from HN1-1400; (b) the binary image of (a), where the white spots indicate the location and the size of nano-clusters. (c) The diameter-distribution of nano-clusters from a); ca. 300 nano-clusters were used for particle size analysis. (d) Relative concentration profiles of $\mathrm{C}, \mathrm{N}, \mathrm{Si}$ and Z-contrast profile across the $X-X^{\prime}$ direction. The scale bars in (a) and an inset of (d) are $20 \mathrm{~nm}$ and $2 \mathrm{~nm}$, respectively.

Figure 5a shows the atomic-resolution Z-contrast STEM image obtained from the 'jellyfish' precipitates found in sample HN1-1400. In the carbon matrix, an extensive number of randomly dispersed individual $\mathrm{Si}$ atoms are identified, which seem to segregate and to form nano-clusters in some regions. Other regions are consisting of aggregated Si-N-based nano-clusters and correspond to the 'jellyfish' contrast observed in the BF-TEM. As some of the clusters exhibit distinct lattice fringes, Fourier transforms (FT) on selected regions (squares in Figure 5a) were performed in order to identify the local structure of the clusters. Although the FT image obtained from the whole area in Figure 5a shows Debye-Scherrer ring pattern (see inset of Figure 5a), we found two types of representative FT images with strong spectral frequencies (Figures $5 \mathrm{~b}-\mathrm{e}$ ). As previously mentioned, the clusters are basically composed of $\mathrm{Si}$ and $\mathrm{N}$ and hence we simulated the SAED patterns of $\alpha-\mathrm{Si}_{3} \mathrm{~N}_{4}$ along the low-index zone axis. Although the FT patterns calculated from the Z-contrast image are relatively distorted, the simulated SAED patterns along the [001] and [111] (or [011]) zoneaxes of $\alpha-\mathrm{Si}_{3} \mathrm{~N}_{4}$ agree well with the experimental FT patterns. Therefore, we conclude that the formed 'jellyfish' precipitates are composed of $\alpha-\mathrm{Si}_{3} \mathrm{~N}_{4}$-like nano-clusters and represent the crystallization onset of the investigated SiCN samples. Based on our findings of the high 
mobility of individual single Si atoms under the electron beam, the crystallization of our SiCN samples is thought to rely on diffusion processes related to the large number of dangling bonds present in the amorphous silicon nitride phase. ${ }^{31-34}$

To our best knowledge, the observed $\alpha-\mathrm{Si}_{3} \mathrm{~N}_{4}$-like nano-clusters are the smallest in size reported so far for silicon nitride-based materials. Thus, a volume of the nano-clusters with a diameter of $0.8 \mathrm{~nm}$ corresponds to that of one unit cell of $\alpha-\mathrm{Si}_{3} \mathrm{~N}_{4}$ or $\mathrm{Si}_{12} \mathrm{~N}_{18},{ }^{35-36}$ i.e. they contain only $12 \mathrm{Si}$ atoms; the larger clusters $(1.5$ and $2.1 \mathrm{~nm})$ consist consequently of 6 and 19 unit cells, respectively (i.e., $\mathrm{Si}_{72} \mathrm{~N}_{108}$ and $\mathrm{Si}_{228} \mathrm{~N}_{342}$, respectively).

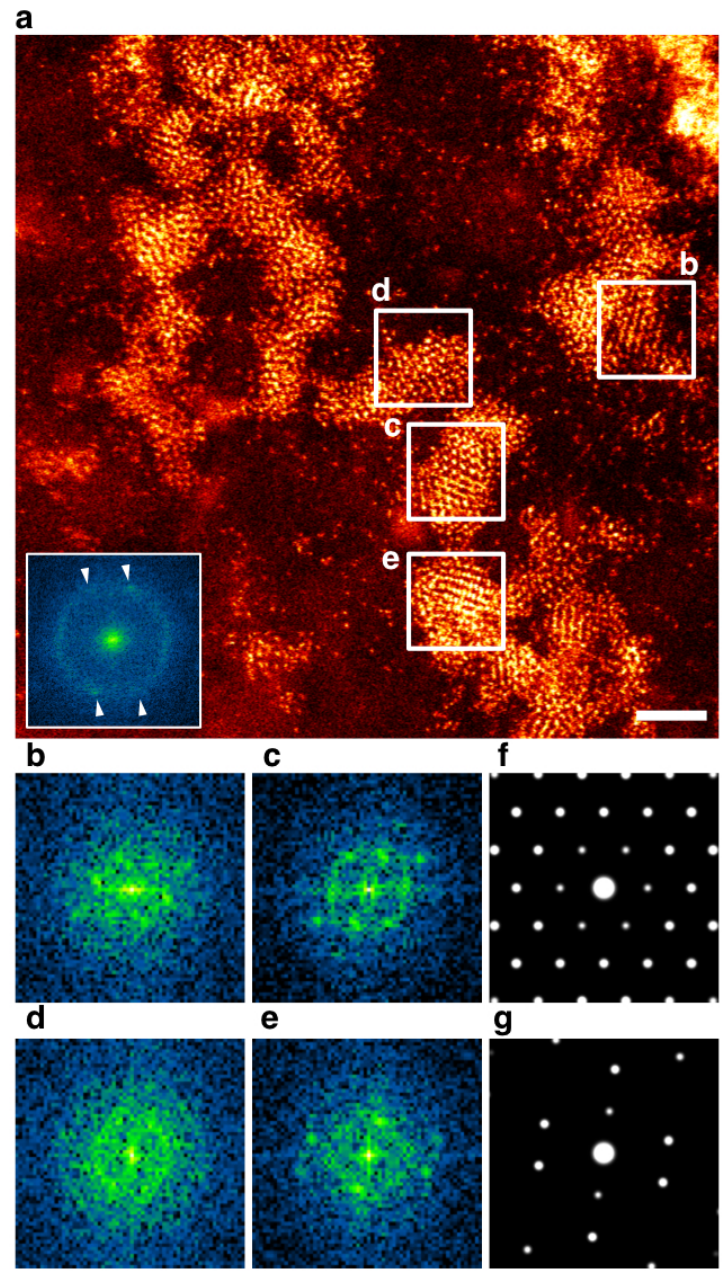

Figure 5. (a) Z-contrast image obtained from the 'jellyfish'-precipitate region of HN1-1400 (inset image represent the two-dimensional Fourier transform taken from the whole image). (b) (e) Two dimensional Fourier transformed taken from the regions marked with (b) - (e) in the image of (a).The simulated SAED patterns of $\alpha-\mathrm{Si}_{3} \mathrm{~N}_{4}$ viewed along the [001] (f) and [111] or [011] axis (g). The scale bar in (a) is $2 \mathrm{~nm}$. 
Metallic and non-metallic clusters attracted increased attention in the last decades, as they address the question which parameters are of relevance for their generation and stability. ${ }^{37}$ Within this context it has been considered that clusters with specific numbers of atoms (i.e., magic-number clusters) exhibit outstanding stability. This has been correlated with closedshell conditions, as shown for rare-gas element clusters ${ }^{38}$ (closed geometric shells, i.e. magic-number clusters show filled icosahedral shells) and metallic clusters ${ }^{39-42}$ (closed electronic shells, i.e., magic numbers determined by the electronic structure). In the case of metallic clusters, the enhanced stability of the magic-number clusters has been also related to their chemical hardness, as shown by Harbola. ${ }^{43}$ While (inter)metallic and non-metallic clusters were intensively studied within the last decades, less is known about clusters from binary and multinary inorganic compounds which exhibit covalent bondings. Thus, only few studies on silica ${ }^{44-48}$ or silicon carbide ${ }^{49,50}$ are known; whereas there is no systematic study related to the formation and stability of silicon nitride clusters. The results presented here can be consequently used to address in future studies the question which parameters determine the formation of stable (magic-number) silicon nitride clusters.

\section{Conclusion}

Polysilylcarbodiimide-derived amorphous SiCN ceramics have been investigated by means of atomic resolution ABF- and ADF-STEM. Contrary to the SiCN sample annealed at 1100 ${ }^{\circ} \mathrm{C}$ which was found to be homogeneous and amorphous (consisting of amorphous silicon nitride and carbon), the sample annealed at $1400{ }^{\circ} \mathrm{C}$ is characterized by a short-rangeordered structure composed of $\alpha-\mathrm{Si}_{3} \mathrm{~N}_{4}$ nanoclusters with average cluster sizes of $0.8,1.5$ and $2.1 \mathrm{~nm}$, embedded in a highly curved-graphene matrix. The sample annealed at $1400^{\circ} \mathrm{C}$ represents the onset of the crystallization in SiCN ceramics, which has been characterized for the first time using atomic-scale STEM techniques. The results obtained within the present study are of fundamental importance for the understanding of the phase separation and crystallization behavior of amorphous and polymer-derived silicon nitride-based materials.

\section{Acknowledgements}

Financial support by the the grants funded under the MWN (Materials World Network) of the DFG and the National Science Foundation as well as LOEWE-Zentrum AdRIA (AdaptronikResearch, Innovation, Application), funded by the state of Hesse, Germany is gratefully 
acknowledged. A part of this study was supported by the Joint Research Project between Japan and Germany through JSPS (Japan Society for the Promotion of Science) Bilateral

Program. R.I. also acknowledges support from JSPS Postdoctoral Fellowship for Research Abroad.

\section{References}

1. Colombo P, Mera G, Riedel R, Soraru GD. Polymer-Derived Ceramics: 40 Years of Research and Innovation in Advanced Ceramics. J Am Ceram Soc 2010; 93 (7): 1805-1837.

2. Schempp S, Durr J, Lamparter P, Bill J, Aldinger F. Study of the atomic structure and phase separation in amorphous Si-C-N ceramics by X-ray and neutron diffraction. Z Naturforsch A 1997; 53 (3-4): 127-133.

3. Durr J, Lamparter P, Bill J, Steeb S, Aldinger F. An X-ray and neutron scattering investigation of precursor derived $\mathrm{Si}_{24} \mathrm{C}_{43} \mathrm{~N}_{33}$ ceramics. J Non-Cryst Solids 1998; 232: 155-161.

4. Uhlig $\mathrm{H}$, Friess $\mathrm{M}$, Lamparter $\mathrm{P}$, Steeb $\mathrm{S}$. Local order of polymer derived amorphous $\mathrm{Si}_{\mathrm{x}} \mathrm{C}_{\mathrm{y}} \mathrm{N}_{\mathrm{z}}$. J Appl Phys 1998; 83 (11): 5714-5718.

5. Mera G, Tamayo A, Nguyen H, Sen S, Riedel R. Nanodomain Structure of Carbon-Rich Silicon Carbonitride Polymer-Derived Ceramics. J Am Ceram Soc 2010; 93 (4): 1169-1175.

6. Kleebe HJ. Microstructure and stability of polymer-derived ceramics; The Si-C-N system. Phys Status Solidi A 1998; 166 (1): 297-313.

7. Seitz J, Bill J, Egger N, Aldinger F. Structural investigations of Si/C/N-ceramics from polysilazane precursors by nuclear magnetic resonance. J Eur Ceram Soc 1996; 16 (8): 885-891.

8. Matsunaga K, Iwamoto Y, Fisher CAJ, Matsubara H. Molecular dynamics study of atomic structures in amorphous Si-C-N ceramics. J Ceram Soc Jpn 1999; 107 (11): 1025-1031.

9. Resta N, Kohler C, Trebin HR. Molecular dynamics Simulations of amorphous Si-C-N ceramics: Composition dependence of the atomic structure. J Am Ceram Soc 2003; 86 (8): 1409-1414.

10. Amkreutz M, Frauenheim T. Understanding precursor-derived amorphous Si-C-N ceramics on the atomic scale. Phys Rev B 2002; 65 (13).

11. Mera G, Navrotsky A, Sen S, Kleebe HJ, Riedel R. Polymer-derived SiCN and SiOC ceramics - structure and energetics at the nanoscale. J Mater Chem A 2013; 1 (12): 3826-3836.

12. Kroke E, Li YL, Konetschny C, Lecomte E, Fasel C, Riedel R. Silazane derived ceramics and related materials. Mat Sci Eng R 2000; 26 (4-6): 97-199.

13. Iwamoto Y, Volger W, Kroke E, Riedel R, Saitou T, Matsunaga K. Crystallization behavior of amorphous silicon carbonitride ceramics derived from organometallic precursors. J Am Ceram Soc 2001; 84 (10): 2170-2178.

14. Andronenko SI, Stiharu I, Misra SK. Synthesis and characterization of polyureasilazane derived SiCN ceramics. J Appl Phys 2006; 99 (11).

15. Andronenko SI, Leo A, Stiharu I, Misra SK. EPR/FMR Investigation of Mn-Doped SiCN Ceramics. Appl Magn Reson 2010; 39 (4): 347-356.

16. Papendorf B, Nonnenmacher K, lonescu E, Kleebe HJ, Riedel R. Strong Influence of Polymer Architecture on the Microstructural Evolution of Hafnium-Alkoxide-Modified Silazanes upon Ceramization. Small 2011; 7 (7): 970-978.

17. Liemersdorf S, Riedel R, Oberle J. Influence of the gas atmosphere on the composition and phase development of polymer-derived SiOC-Ceramics. J Am Ceram Soc 2008; 91 (1): 325-328. 
18. Gao Y, Mera G, Nguyen H, Morita K, Kleebe HJ, Riedel R. Processing route dramatically influencing the nanostructure of carbon-rich $\mathrm{SiCN}$ and $\mathrm{SiBCN}$ polymer-derived ceramics. Part I: Low temperature thermal transformation. J Eur Ceram Soc 2012; 32 (9): 1857-1866.

19. Ferrari AC, Robertson J. Interpretation of Raman spectra of disordered and amorphous carbon. Phys Rev B 2000; 61 (20): 14095-14107.

20. Cancado LG, Takai K, Enoki T, Endo M, Kim YA, Mizusaki H, Jorio A, Coelho LN, MagalhaesPaniago R, Pimenta MA. General equation for the determination of the crystallite size L-a of nanographite by Raman spectroscopy. Appl Phys Lett 2006; 88 (16).

21. Lucchese MM, Stavale F, Ferreira EHM, Vilani C, Moutinho MVO, Capaz RB, Achete CA, Jorio A. Quantifying ion-induced defects and Raman relaxation length in graphene. Carbon 2010; 48 (5): 1592-1597.

22. Widgeon SJ, Sen S, Mera G, lonescu E, Riedel R, Navrotsky A.Si-29 and C-13 Solid-State NMR Spectroscopic Study of Nanometer-Scale Structure and Mass Fractal Characteristics of Amorphous Polymer Derived Silicon Oxycarbide Ceramics. Chem Mater 2010; 22 (23): 6221-6228.

23. Widgeon S, Mera G, Gao Y, Stoyanov E, Sen S, Navrotsky A, Riedel R. Nanostructure and Energetics of Carbon-Rich SiCN Ceramics Derived from Polysilylcarbodiimides: Role of the Nanodomain Interfaces. Chem Mater 2012; 24 (6): 1181-1191.

24. Findlay SD, Shibata N, Sawada H, Okunishi E, Kondo Y, Yamamoto T, Ikuhara Y. Robust atomic resolution imaging of light elements using scanning transmission electron microscopy. Applied Physics Letters 2009; 95: 191913.

25. Huang R, Ikuhara YH, H, Mizoguchi T, Findlay S, D, Kuwabara A, Fisher C, A, J, Moriwake H, Oki H, Hirayama T, Ikuhara Y. Oxygen-Vacancy Ordering at Surfaces of Lithium Manganese (III,IV) Oxide Spinel Nanoparticles. Angew. Chem. Int. Ed. 2011; 50: 3053-3057.

26 Yu H, Ishikawa R, So YG, Shibata N, Kudo T, Zhou H, Ikuhara Y,. Direct Atomic-Resolution Observation of Two Phases in the $\mathrm{Li}_{1.2} \mathrm{Mn}_{0.567} \mathrm{Ni}_{0.166} \mathrm{Co}_{0.067} \mathrm{O}_{2}$ Cathode Material for Lithium-Ion Batteries. Angew. Chem. Int. Ed. 2013; 52: 1-6.

27 Findlay SD, Saito T, Shibata N, Sato Y, Matsuda J, Asano K, Akiba E, Hirayama T, Ikuhara Y. Direct Imaging of Hydrogen within a Crystalline Environment. Applied Physics Express 2010; 3: 116603.

28 Ishikawa R, Okunishi E, Sawada H, Kondo Y, Hosokawa F, Abe E. Direct imaging of hydrogen-atom columns in a crystal by annular bright-field electron microscopy. Nature Mater 2011; 10: $278-281$.

29. Pennycook SJ, Boatner LA. Chemically sensitive structure-imaging with a scanning transmission electron microscope. Nature 1998; 336 (6199): 565-567.

30. Schneider CA, Rasband WS, Eliceiri KW; NIH Image to ImageJ: 25 years of image analysis. Nat Meth 2012; 9 (7): 671-675.

31. Seher M, Bill J, Aldinger F, Riedel R. Crystallization Kinetics of Polysilazane-Derived Amorphous-Silicon Nitride. J Cryst Growth 1994; 137 (3-4): 452-456.

32. Li YH, Wang L, Yin SW, Yang FM, Wu P. Rapid Crystallization Process of Amorphous Silicon Nitride. J Am Ceram Soc 2011; 94 (12): 4169-4173.

33. Tsuruta K, Omeltchenko A, Kalia RK, Vashishta P. Early stages of sintering of silicon nitride nanoclusters: A molecular-dynamics study on parallel machines. Europhys Lett 1996; 33 (6): 441-446.

34. Kalia RK, Nakano A, Tsuruta K, Vashishta P. Morphology of pores and interfaces and mechanical behavior of nanocluster-assembled silicon nitride ceramic. Phys Rev Lett 1997; 78 (4): 689-692.

35. Kato K, Inoue Z, Kijima K, Kawada I, Tanaka H,Yamane T. Structural Approach to Problem of Oxygen-Content in Alpha Silicon-Nitride. J Am Ceram Soc 1975; 58 (3-4): 90-91.

36. Toraya H. Quantitative phase analysis of alpha- and beta-silicon nitrides. I. Estimation of errors. J Appl Crystallogr 1999; 32: 704-715.

37. Castelman AW, Bowen KH. Clusters: Structure, Energetics and Dynamics of Intermediate States of Matter. J Phys Chem 1996; 100(31): 12911-12944. 
38. Echt O, Sattler K, Recknagel E. Magic Numbers for Sphere Packings: Experimental Verification in Free Xenon Clusters. Phys Rev Lett 1981; 47: 1121.

39. Knight WD, Clemenger K, de Heer WA, Saunders WA, Chou MY, Cohen ML. Electronic Shell Structure and Abundances of Sodium Clusters. Phys Rev Lett 1984; 52: 2141.

40. Pyykkö P. Magic Nanoclusters of Gold. Nature Nanotechnol 2007; 2: 273-274.

41. Schmid G, Fenske D. Metal clusters and Nanoparticles. Phil Trans R Soc 2010; 368: 20171210.

42. Desireddy A, Kumar S, Guo J, Bolan MD, Griffith WP, Bigioni TP. Temporal Stability of Magicnumber Metal Clusters: Beyond the Shell Closing Model. Nanoscale 2013; 5: 2035-2044.

43. Harbola MK. Magic numbers for metallic clusters and the principle of maximum hardness. Proc Nat Acad Sci USA 1992; 89: 1036-1039.

44. Anderson JS, Ogden JS. Matrix Isolation Studies of Group-IV Oxides. I. Infrared Spectra and Structures of $\mathrm{SiO}, \mathrm{Si}_{2} \mathrm{O}_{2}$, and $\mathrm{Si}_{3} \mathrm{O}_{3}$. J Chem Phys 1969; 51:4189-4196.

45. Jeong S, Fisher KJ, Howe RF, Willett GD. Laser ablation Fourier transform mass spectrometric study of zeolites. Microporous Mater 1995; 4: 467-473.

46. Fan J, Nicholas JB, Price JM, Colson SD, Wang LS. $\mathrm{Si}_{3} \mathrm{O}_{4}{ }^{-}$: vibrationally resolved photoelectron spectrum and ab initio calculations. J Am Chem Soc 1995; 117: 5417-5418.

47. Wang LS, Desai SR, Wu H, Nicholas JB. Small silicon oxide clusters: chains and rings. Z Phys D 1997; 40: 36-39.

48. Xu C, Wang W, Zhang W, Zhuang J, Liu L, Kong Q, Zhao L, Long Y, Fan K, Qian S, Li S. Magic Numbers in Silicon Dioxide-Based Clusters. J Phys Chem A 2000; 104: 9518-9524.

49. Ray AK, Huda MN. Silicon-Carbide Nanoclusters: A Pathway to Future Nanoelectronics. J Comp Theor Nanosci 2006; 3(3): 315-341.

50. Melinon P, Masenelli B, Tournus F, Perez A. Playing with carbon and silicon at the nanoscale. Nature Mater 2007; 6: 479-490.

\section{Figure Captions}

Figure 1. (a) X-ray diffraction patterns and (b) Micro-Raman spectra obtained from HN1-1100 and HN1-1400, respectively.

Figure 2. Radial integrated profiles (a) derived from SAED patterns obtained from (b) HN1-1100 and (c) HN1-1400. The indices of the Debye-Scherrer rings (arrowheads) correspond to the 110 and 120 reflections of graphite, respectively. BF-TEM images obtained from HN1-1100 (d) and HN1-1400 (e); the scale bar in all images is $100 \mathrm{~nm}$.

Figure 3. Simulatenously recorded ABF/ADF-STEM images obtained from HN1-1100 ( $a$ and $b$, resprectively) and HN1-1400 (c and d, respectively). The scale of the images (a) - (d) is the same and the scale bar in (a) is $5 \mathrm{~nm}$. The white-square regions are enlarged in the inset images (the scale bar in the insets is $2 \mathrm{~nm}$ ).

Figure 4. (a) Low-magnification Z-contrast image obtained from HN1-1400; (b) the binary image of (a), where the white spots indicate the location and the size of nano-clusters. (c) The diameter-distribution of nano-clusters from a); ca. 300 nano-clusters were used for particle size analysis. (d) Relative concentration profiles of $\mathrm{C}, \mathrm{N}, \mathrm{Si}$ and Z-contrast profile across the $X-X^{\prime}$ direction. The scale bars in (a) and an inset of (d) are $20 \mathrm{~nm}$ and $2 \mathrm{~nm}$, respectively.

Figure 5. (a) Z-contrast image obtained from the 'jellyfish'-precipitate region of HN1-1400 (inset image represent the two-dimensional Fourier transform taken from the whole image). (b) (e) Two dimensional Fourier transformed taken from the regions marked with (b) - (e) in the image of (a).The simulated SAED patterns of $\alpha-\mathrm{Si}_{3} \mathrm{~N}_{4}$ viewed along the [001] (f) and [111] or [011] axis (g). The scale bar in (a) is $2 \mathrm{~nm}$. 